\title{
A LIE PROPERTY IN GROUP RINGS
}

\author{
ANTONIO GIAMBRUNO AND SUDARSHAN K. SEHGAL
}

(Communicated by Donald S. Passman)

\begin{abstract}
Let $A$ be an additive subgroup of a group ring $R$ over a field $K$. Denote by $[A, R]$ the additive subgroup generated by the Lie products $[a, r]=a r-r a, a \in A, r \in R$. Inductively, let $\left[A, R_{n}\right]=\left[\left[A, R_{n-1}\right], R\right]$. We prove that $\left[A, R_{n}\right]=0$ for some $n \Rightarrow[A, R] R$ is a nilpotent ideal.
\end{abstract}

\section{INTRODUCTION}

Let $A$ be an additive subgroup of a ring $R$. We define $[A, R]$ to be the additive subgroup of $R$ generated by all Lie products $[a, r]=a r-r a, a \in A$, $r \in R$. Inductively, we set

$$
[A, \underbrace{R, R, \ldots, R}_{n+1}]=\left[A, R_{n+1}\right]=\left[\left[A, R_{n}\right], R\right] .
$$

The main result of this note is

Theorem. Let $R=K G$ be the group algebra of a group $G$ over a field $K$ and $A$ an additive subgroup of $R$. Then $\left[A, R_{n}\right]=0$ for some $n \Rightarrow[A, R] R$ is an (associative) nilpotent ideal of $R$.

Taking $A=K G$, we conclude that if $K G$ is Lie nilpotent, then

$$
[K G, K G] K G=\Delta\left(G^{\prime}\right) K G
$$

is nilpotent where $\Delta(G)$ is the augmentation ideal of the derived group $G$. This implies that $G$ is abelian if $\chi(K)$, the characteristic of $K$ is 0 and $G$ is a finite $p$-group if $\chi(K)=p>0$. This is a theorem of Passi, Passman and Sehgal $[3,5$, p. 151]. To prove our theorem we need two results due to Gupta, Levin and Passman.

Lemma 1 [1]. The nth term $G_{n}$ of the lower central series of $G$ is contained in

$$
1+[\underbrace{R, R, \ldots, R}_{n}] R \quad \text { where } R=K G .
$$

Received by the editors December 2, 1987 and, in revised form, February 22, 1988.

1980 Mathematics Subject Classification (1985 Revision). Primary 16A27, 16A68.

This research is supported by NSERC, Canada and MPI, Italy. 
Lemma 2 [4, p. 202]. If $K G$ satisfies a nondegenerate multilinear generalized polynomial identity then $\phi$, the $F C$ subgroup of $G$, is of finite index and has finite derived group $\phi^{\prime}$.

In the next section we collect some preliminary results and prove the theorem in $\S 3$.

\section{SOME PRELIMINARY RESULTS}

In this section $R$ is an arbitrary ring with 1 and $A$ is an additive subgroup of $R$. We denote by $C_{R}(A)=\{x \in R \mid x a=a x$ for all $a \in A\}$, the centralizer of $A$ in $R$. We write $[x, y, z]=[[x, y], z]$ for $x, y, z \in R$.

Lemma 3. (i) For $k \geq 1,\left[A, R_{k}\right] R=R\left[A, R_{k}\right]$ so that $\left[A, R_{k}\right] R$ is the twosided ideal generated by $\left[A, R_{k}\right]$.

(ii) For $a, b, x, y \in R$ we have

$$
[a, b y, x]=[a, b, x] y+[a, b][y, x]+b[a, y, x]+[b, x][a, y] .
$$

(iii) $[R, R, R][A, R] \leq[A, R, R] R$.

(iv) For $b \in C_{R}(A),[A, R][b, R] \leq[A, R, R] R$.

Proof. (i) Let $a \in\left[A, R_{k-1}\right], x, y \in R$. Then

$$
[a, x] y=(a x-x a) y=a(x y)-(x y) a-x(a y-y a)=[a, x y]-x[a, y]
$$

and

$$
x[a, y]=[a, x y]-[a, x] y .
$$

(ii) is clear.

To obtain (iii) in the expression for $[b, x][a, y]$ in (ii), we take $a \in A$, $b \in[R, R], x, y \in R$ to conclude

$$
\begin{aligned}
{[R, R, R][A, R] } & \leq[A, R, R] R+[A,[R, R]] R+R[A, R, R] \\
& \leq[A, R, R] R \quad(\text { by the Jacobi identity and (i)). }
\end{aligned}
$$

(iv) We first observe that for $a \in A, r \in R,[a, r] b=[a, r b]$ and thus $[A, R] b \leq[A, R]$. Now, taking $x \in[A, R], r \in R$ we have

$$
x[b, r]=[x b, r]-[x, r] b
$$

which implies

$$
[A, R][b, R] \leq[[A, R] b, R]+[A, R, R] R \leq[A, R, R] R .
$$

The next lemma is an extension of a result of Jennings [2] to our situation. The proof parallels that of Jennings.

Lemma 4. Suppose that $\left[A, R_{n}\right]=0$ for some $n \geq 1$. Then $[A, R] R$ is a nil ideal and $[A, R, R] R$ is a nilpotent ideal.

Proof. We first claim

$$
\text { If }\left[A, R_{n}\right]=0 \text { for some } n>2 \text { then }\left(\left[A, R_{n-1}\right] R\right)^{2}=0 \text {. }
$$


Let $u, v \in\left[A, R_{n-3}\right]$ and $x, y \in R$. Then by the Jacobi identity

$$
\begin{aligned}
-[[u, x],[v, y]] & =[v,[y,[u, x]]]+[y,[[u, x], v]] \\
& =[[u, x], y, v]-[[u, x], v, y] \in\left[A, R_{n}\right]=0 .
\end{aligned}
$$

This means that $\left[A, R_{n-2}\right]$ is commutative. Now, pick $a, b \in\left[A, R_{n-2}\right]$, $x, y \in R$, and use Lemma 3(ii) to conclude

$$
[a, b y, x]=[a, b, x] y+b[a, y, x]+[b, x][a, y] .
$$

Since $\left[A, R_{n}\right]=0$, we obtain $[b, x][a, y]=0$ i.e. $\left(\left[A, R_{n-1}\right]\right)^{2}=0$. Due to the fact that $\left[A, R_{n-1}\right]$ is central we may conclude that $\left(\left[A, R_{n-1}\right] R\right)^{2}=0$ establishing our claim $(*)$.

To prove our lemma by induction on $n$, we may assume that $n \geq 2$. First let $n=2$ i.e. $[A, R, R]=0$. We have to show that $[A, R] R$ is a nil ideal. Since $[A, R]$ is central it is enough to prove that $[A, R]$ is nil. In Lemma 3(ii), take $a \in A, x \in R, x=y, a=b$; we get $[a, x]^{2}=0$, proving the lemma for $n=2$. Now, let $n>2$. Writing $\bar{R}=R /\left[A, R_{n-1}\right] R$ and $\bar{A}$ for the homomorphic image of $A$ etc., we have $\left[\bar{A}, \bar{R}_{n-1}\right]=0$. Therefore, due to the induction hypothesis $[\bar{A}, \bar{R}] \bar{R}$ is nil and $[\bar{A}, \bar{R}, \bar{R}] \bar{R}$ is nilpotent. Hence due to $(*)$ we conclude that $[A, R] R$ is nil and $[A, R, R] R$ is nilpotent as desired.

Remark. The statement of our theorem is not true for arbitrary rings. This was conjectured by Jennings [2] and confirmed by Gupta and Levin [1] by means of an example. We record another easy example to the same effect.

Example. Let $E$ be the exterior algebra on a countable infinite-dimensional vector space $V$ over a field of characteristic not 2 . Let

$$
V=\operatorname{Span}\left\{v_{1}, v_{2}, \ldots\right\}, \quad E=\operatorname{Span}\left\{v_{i_{1}} v_{i_{2}} \cdots v_{i_{m}} \mid i_{1}<i_{2}<\cdots<i_{m}\right\} .
$$

We have $[E, E]=\operatorname{Span}\left\{v_{i_{1}} v_{i_{2}} \cdots v_{i_{2 m}} \mid i_{1}<i_{2}<\cdots<i_{2 m}\right\}$ and $[E, E, E]=0$. It is clear that $[E, E]$ has elements of arbitrarily large index of nilpotency; for instance, $a=v_{1} v_{2}+v_{3} v_{4}+\cdots+v_{2 m-1} v_{2 m}$ has index of nilpotency $m+1$.

\section{Proof of theorem}

In this section we shall fix $R=K G, A$ the given additive subgroup of $R$, $\phi$ the FC-subgroup of $G$. We shall denote by $\Delta(G, N)=(\Delta N) K G$ the kernel of the homomorphism $K G \rightarrow K(G / N)$ where $\Delta(N)$ is the augmentation ideal of the normal subgroup $N$. We separate a special case as

Lemma 5. Suppose that $\phi$ the FC-subgroup of $G$ is abelian and of finite index. Then $\left[A, R_{n}\right]=0$ for some $n \Rightarrow[A, R]=0$.

Proof. Let $m=(G: \phi)$. Then fix a right transversal $\left\{1=x_{1}, x_{2}, \ldots, x_{m}\right\}$ of $\phi$ in $G$. Write $\left(\phi, x_{i}\right)=\left\{a^{-1} x_{i}^{-1} a x_{i} \mid a \in \phi\right\}$ and $S_{i}=\Delta\left(\phi, x_{i}\right)$ for $i=$ $2, \ldots, m$ and $S=S_{2} S_{3} \cdots S_{m}$. It is well known [5, p. 145, 4 or 3] that there is an imbedding $\rho: K G \rightarrow M_{m}(K \phi)$ of $K G$ into $m \times m$ matrices over the 
commutative group ring $K \phi$. Moreover, since $K \phi$ imbeds in $M_{m}(K \phi)$ as scalar matrices,

$$
M_{m}(K \phi) \geq K \phi \cdot \rho(K G) \geq M_{m}(S) .
$$

We may clearly assume that $A \neq 0$ so that $K \phi \rho(A) \neq 0$. Since $K \phi$ are scalar matrices, we have

$$
[K \phi \rho(A), K \phi \rho(K G), \ldots, K \phi \rho(K G)]=(K \phi)^{n} \rho([A, K G, K G, \ldots, K G])=0 \text {. }
$$

Let $0 \neq u \in \rho(A)$ and write $u=\sum_{i, j} x_{i j} e_{i j}$ with $x_{i j} \in K \phi$, where the $e_{i j}$ are the usual matrix units. Let $i \neq j$ be fixed; then for $s_{1}, \ldots, s_{n} \in S$ we have

$$
\begin{aligned}
0 & =\left[u, s_{1} e_{i i}, s_{2} e_{j j}, s_{3} e_{j j}, \ldots, s_{n} e_{j j}\right] \\
& =s_{1} s_{2} \cdots s_{n}\left[\sum_{l=1}^{n} x_{l i} e_{l i}-\sum_{t=1}^{n} x_{i t} e_{i t}, e_{j j}, \ldots, e_{j j}\right] \\
& =\cdots= \pm s_{1} s_{2} \cdots s_{n} x_{i j} e_{i j} \pm s_{1} s_{2} \cdots s_{n} x_{j i} e_{j i} .
\end{aligned}
$$

Thus $s_{1} s_{2} \cdots s_{n} x_{i j}=0$ and since $s_{i}$ are arbitrary elements of $S$ which is a product of augmentation ideals of infinite groups, it follows that $x_{i j}=0$ for all $i \neq j$. Thus $u=\sum_{i=1}^{m} x_{i i} e_{i i}$. Suppose that $x_{l l} \neq 0$. Then for $j \neq l$, we get

$$
\begin{aligned}
0 & =\left[u, s_{1} e_{l j}, s_{2} e_{l l}, \ldots, s_{n} e_{l l}\right]=s_{1} s_{2} \cdots s_{n}\left[\left(x_{l l}-x_{j j}\right) e_{l j}, e_{l l}, \ldots, e_{l l}\right] \\
& =s_{1} s_{2} \cdots s_{n}\left(x_{l l}-x_{j j}\right) e_{l j} .
\end{aligned}
$$

This implies that $s_{1} s_{2} \cdots s_{n}\left(x_{l l}-x_{j j}\right)=0$ for all $s_{i} \in \Delta\left(\phi, x_{i}\right)$. It follows that $x_{l l}=x_{j j}$ for all $j=1,2, \ldots, m$. Thus $u$ is a scalar matrix. Hence, $0=[\rho(A), \rho(K G)]=\rho[A, K G]$ and so $[A, K G]=0$.

We are now ready to give the

Proof of the Theorem. We know by Lemma 4 that $[A, R] R$ is a nil ideal and $[A, R, R] R$ is a nilpotent ideal. If $\chi(K)=0, R$ contains no nonzero nil ideals $[4$, p. 47]; so $[A, R] R=0$ and we are done. Therefore, we suppose that $\chi(K)=$ $p>0$. If $G_{3}$ is the third term of the lower central series of $G$ then by Lemma ' 1 we have $\Delta\left(G_{3}\right) \leq[R, R, R] R$ and so it follows that $\Delta\left(G, G_{3}\right)=\Delta\left(G_{3}\right) R \leq$ $[R, R, R] R$. Since by Lemma 3(iii), $[R, R, R] R[A, R]=[R, R, R][A, R] R \leq$ $[A, R, R] R$ we get

$$
\Delta\left(G_{3}\right) R[A, R] \leq[A, R, R] R
$$

which is a nilpotent ideal. So $\Delta\left(G_{3}\right) R[A, R]$ is a nilpotent ideal of $R$.

Now, let $\pi: K G \rightarrow K\left(G / G_{3}\right)$ be the natural map. If the theorem holds for nilpotent class two groups, then, we will have

$$
\pi([A, R] R)=[\pi(A), \pi(R)] \pi(R) \text { is a nilpotent ideal. }
$$

So for some $m \geq 1,([A, R] R)^{m} \leq \Delta\left(G_{3}\right) R$ and

$$
([A, R] R)^{m}[A, R] \leq \Delta\left(G_{3}\right) R[A, R], \quad \text { a nilpotent ideal. }
$$

So $([A, R] R)^{m+1}$ and hence $[A, R] R$ is a nilpotent ideal, the desired conclusion. Thus we may assume that $G$ is nilpotent of class two, and so, $G^{\prime}$ is 
central. Since $\left[A, R_{n}\right]=0$, for $a \in A,\left[a, x_{1}, \ldots, x_{n}\right]$ is a multilinear generalized polynomial identity for $R$. Also, if for all $a \in A,\left[a, x_{1}\right] x_{2} \cdots x_{n}$ is a generalized polynomial identity for $R$, then since $1 \in R$, we get $[A, R]=0$. The theorem is proved in this case. Thus, we may assume that for some $a \in A$, $\left[a, x_{1}, \ldots, x_{n}\right]$ is a nondegenerate generalized polynomial identity for $R$. It follows by Lemma 2 that $(G: \phi)<\infty$ and $\left|\phi^{\prime}\right|<\infty$. Let $P$ be the Sylow $p$-subgroup of $\phi^{\prime}$. Since $\Delta(G, P)$ is nilpotent, factoring by $P$, it is enough to prove the theorem when $\phi^{\prime}$ is a $p^{\prime}$-group. We use induction on $\left|\phi^{\prime}\right|$. If $\left|\phi^{\prime}\right|=1, \phi$ is abelian and we are done by Lemma 5 . Thus we assume $\left|\phi^{\prime}\right|>1$. Since $\left[A, R_{n}\right]=0$, then also $\left[A, R_{p^{m}}\right]=0$ where $p^{m}$ is the smallest power of $p$ greater than $n$. Thus for $x \in R, a \in A$, we get

$$
0=[a, \underbrace{x, x, \ldots, x}_{p^{m}}]=a x^{p^{m}}-x^{p^{m}} a \text {. }
$$

Therefore, for all $x \in R, x^{p^{m}} \in C_{R}(A)$. Now, if for all $g \in \phi, g^{p^{m}}$ belongs to the centre of $\phi$ then it follows by Schur's theorem [5, p. 39] that $\phi^{\prime}$ is a $p$-group contrary to our assumption that $\phi^{\prime}$ is a nontrivial $p^{\prime}$-group. Thus there is a $g \in \phi$ such that $g^{p^{m}} \notin C(\phi)$, the centre of $\phi$. Pick $h \in \phi$ such that $g^{p^{m}} h-$ $h g^{p^{m}} \neq 0$. Since $g^{p^{m}} \in C_{R}(A)$, we have, by Lemma 3(iv), $[A, R]\left[g^{p^{m}}, h\right] \leq$ $[A, R, R] R$. Thus letting $z=h^{-1} g^{-p^{m}} h g^{p^{m}}$ we get

$$
\begin{aligned}
{[A, R] R(1-z) } & =[A, R] R h^{-1} g^{-p^{m}} g^{p^{m}} h(1-z)=[A, R] R\left[g^{p^{m}}, h\right] \\
& =R[A, R]\left[g^{p^{m}}, h\right] \leq[A, R, R] R .
\end{aligned}
$$

Since the last ideal is nilpotent by Lemma 4 , we have that $[A, R] R(1-z)$ is a nilpotent ideal. Since $z$ is central in $G, G$ being nilpotent of class two, we have

$$
([A, R] R)^{k}(1-z)^{k}=0 \text { for some } k \geq 1 .
$$

Suppose $k>1$ and let $l_{R}(1-z)$ be the left annihilator of $(1-z)$ in $R$. Then

$$
([A, R] R)^{k}(1-z)^{k-1} \in R(1-z) \cap l_{R}(1-z)=R(1-z) \cap R \hat{z}=0,
$$

$\hat{z}=\left(1+z+\cdots+z^{|z|-1}\right)$. By iterating this process we conclude that

$$
([A, R] R)^{k}(1-z)=0 .
$$

Factoring $G$ by $\langle z\rangle$ we conclude by the inductive hypothesis that

$$
([A, R] R)^{t} \leq \Delta(G,\langle z\rangle)=R(1-z) .
$$

Therefore, $([A, R] R)^{k t}(1-z)=0$ and $([A, R] R)^{k t} \leq R(1-z)$. It follows that $([A, R] R)^{k t} \leq R(1-z) \cap R \hat{z}=0$ as desired.

\section{REFERENCES}

1. N. Gupta and F. Levin, On the Lie ideals of a ring, J. Algebra 81 (1983), 225-231.

2. S. A. Jennings, On rings whose associated Lie rings are nilpotent, Bull. Amer. Math. Soc. 53 (1947), 593-597. 
3. I. B. S. Passi, D. S. Passman and S. K. Sehgal, Lie solvable group rings, Canad. J. Math. 25 (1973), 748-757.

4. D. S. Passman, The algebraic structure of group rings, Wiley, New York, 1977.

5. S. K. Sehgal, Topics in group rings, Marcel Dekker, New York, 1978.

Department of Mathematics, University of Palermo, Palermo, Italy

Department of Mathematics, University of Alberta, Edmonton, Canada 